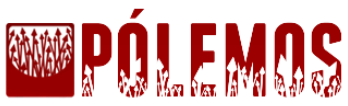

\section{A EQUIVALÊNCIA ENTRE MASSA E ENERGIA ${ }^{\mathbf{1}}$}

Publicado pela primeira vez em 12 de setembro de 2001; revisão substancial em 6 de fevereiro de 2012

\section{Raphael Santos Lapa (Tradutor)}

Graduando em Filosofia pela Universidade de Brasília - UnB

The following is the translation of Professor Fernflores's entry in the Stanford Encyclopedia of Philosophy . The translation follows the version of the entry in the SEP archives at [ http://plato.stanford.edu/archives/spr2012/entries/equivME/ ]. This translated version may differ from the current version of the entry, which may have been updated since the time of this translation. The current version is located at [ http://plato.stanford.edu/entries/equivME/ ]. We'd like to thank the Editors of the Stanford Encyclopedia of Philosophy for granting permission to translate and to publish this entry on the web.

Einstein descreveu corretamente a equivalência entre massa e energia como "o resultado mais importante da teoria da relatividade restrita” (Einstein, 1919), pois este resultado está no cerne da física moderna. De acordo com a famosa equação de Einstein $E=$ $m c^{2}$, a energia $E$ de um sistema físico é numericamente igual ao produto de sua massa $m$ e da velocidade da luz $c$ ao quadrado. É costumeiro referir-se a este resultado como "a equivalência entre massa e energia", ou simplesmente "equivalência massa-energia", porque pode-se escolher unidades nas quais $c=1 \mathrm{e}$, portanto, $E=m$.

As duas principais questões filosóficas que cercam a equação de Einstein referem-se a como devemos entender a afirmação de que massa e energia são, em algum sentido, equivalentes e como devemos entender afirmações sobre a conversibilidade de massa em energia (ou vice-versa).

Neste artigo, primeiro discutiremos a física de $E=m c^{2}$ e sua aplicação (Seção 1). Na Seção 2, identificamos seis interpretações filosóficas distintas, embora relacionadas, da equação de Einstein. Discutiremos, então, na Seção 3 , a história das derivações de $E=m c^{2}$ e sua importância filosófica. Finalmente, na Seção 4 fazemos um relato seletivo da confirmação empírica da equação de Einstein, que se concentra na primeira confirmação de Cockcroft \& Walton (1932) de equivalência massa-energia e numa muito recente e precisa confirmação dada por Rainville et al. (2005).

- $\quad$. A Física de $\mathrm{E}=\mathrm{mc}^{2}$

○ 1.1 Uma interpretação mínima de $\mathrm{E}=\mathrm{mc}^{2}$

\footnotetext{
${ }^{1}$ Agradeço imensamente à disponibilidade do Prof. Dr. Osvaldo Pessoa (USP) pelos comentários, sugestões e revisão na tradução do referido artigo.
} 
- $\quad \underline{1.2 \text { Exemplos de } \mathrm{E}=\mathrm{mc}^{2} \text { em funcionamento }}$

- 1.2.1 Massa e energia de um único corpo

- 1.2.2 Massa e energia de um gás ideal

- 1.2.3 Massa e energia em colisões de partículas pontuais

- 2. Interpretações filosóficas de $\mathrm{E}=\mathrm{mc}^{2}$

- 2.1 Equívocos sobre $\mathrm{E}=\mathrm{mc}^{2}$

- 2.2 Interpretações de mesma-propriedade de $\mathrm{E}=\mathrm{mc}^{2}$

- 2.3 Interpretações de diferentes-propriedades de $\mathrm{E}=\mathrm{mc}^{2}$

- 2.3.1 A interpretação de diferentes-propriedades e não-conversão de Bondi \& Spurgin,

- $\quad$ 2.3.2 A interpretação de diferentes-propriedades e conversão de Rindler

- 2.3.3 A interpretação de uma-propriedade e não-conversão de Lange

- 2.4 Interpretações de $\mathrm{E}=\mathrm{mc}^{2}$ e hipóteses sobre a natureza da matéria

- 2,5 Interpretações ontológicas de $\mathrm{E}=\mathrm{mc}^{2}$

- $\quad$ 3. História das derivações de equivalência massa-energia

- $\quad 3.1$ Derivações de $\mathrm{E}=\mathrm{mc}^{2}$ que usam a teoria de Maxwell

- 3.2 Derivações puramente dinâmicas de $\mathrm{E}=\mathrm{mc}^{2}$

- 4. Verificação experimental da equivalência massa-energia

- $\quad$ 5. Conclusão

- $\quad$ Bibliografia

\section{A Física de $E=m c^{2}$}

Nesta seção, apresentamos primeiramente uma interpretação mínima de $E=m c^{2}$ (na Seção 1.1). A interpretação é mínima no sentido de que faz tão poucos compromissos metafísicos e ontológicos quanto possível. Além disso, é uma interpretação com a qual quase todos os físicos e filósofos agora concordam. Em seguida, ilustraremos as implicações físicas da equação de Einstein considerando três exemplos típicos de equivalência massa-energia em funcionamento (na Seção 1.2). Discutimos a interpretação filosófica de $E=m c^{2}$ separadamente logo depois (na Seção 2 ).

\subsection{Uma interpretação mínima de $E=m c^{2}$}

Para interpretar $E=m c^{2}$ precisamos primeiro entender o significado dos símbolos $E$ e $m$. Infelizmente, esses símbolos não são usados univocamente por físicos e filósofos. No entanto, uma interpretação comum, que adotaremos por enquanto, é que $E$ representa a energia total de um sistema físico $S$. O símbolo $m$ representa a massa relativística de $S$, que é a massa de $S$, medida por um observador $O$ que se move com uma velocidade constante $v$ em relação a $S$. 
No caso especial em que $O$ e $S$ estão num estado de repouso relativo, a massa de $S$ medida por $O$ é chamada de massa de repouso, muitas vezes designada por $m_{o}$. A massa de repouso de $S$ é uma medida da inércia de $S$, isto é, da tendência de $S$ para resistir às mudanças na velocidade. Assim, a massa de repouso de $S$ é simplesmente a sua massa inercial. A massa de repouso está relacionada com a massa relativística pela equação:

$$
m=m_{o} \gamma(v)
$$

onde $\gamma(v)=\left(1-v^{2} / c^{2}\right)^{-1 / 2}$ é o conhecido fator de Lorentz.

O valor da energia que obtemos da equação de Einstein quando $S$ e $O$ estão em um estado de repouso relativo é muitas vezes chamado de energia de repouso e é comumente designado $E_{0}$. Significativamente, Einstein muitas vezes chamou a energia de repouso de o “conteúdo de energia [Energieinhalt]" (1905b) do corpo, uma vez que é uma medida de toda a energia, incluindo a energia potencial, dos constituintes de $S$.

Podemos exibir as relações entre as várias massas e energias que discutimos ao escrever a equação de Einstein da seguinte forma:

$$
E=m_{o} \gamma(v) c^{2}
$$

onde nós simplesmente substituímos a expressão para a massa relativística de (1) em $E=m c^{2}$. No referencial de repouso de $S$ o fator de Lorentz é 1 , porque a velocidade $v$ de $S$ em relação a seu próprio referencial de repouso é zero. Consequentemente, quando $O$ e $S$ estão em um estado de repouso relativo, a equação (2) torna-se:

$$
E_{o}=m_{o} c^{2}
$$

onde escrevemos $E_{o}$ no lado esquerdo de (므) para indicar que estamos nos referindo à energia de repouso de $S$.

Faremos, de agora em diante, referência exclusivamente ao resultado expresso por (므). Consequentemente, simplesmente chamaremos $E_{o}$ de "a energia de $S$ " e $m_{o}$ de "a massa de $S$ ", a menos que precisemos qualificar essas expressões para evitar ambiguidade ou para enfatizar uma característica particular de algum resultado. Além disso, vamos seguir a prática bastante comum na literatura física de abandonar o subscrito " $o$ " de $E_{o}$ e $m_{o}$, respectivamente. Assim, a 
partir de agora, vamos usar $E$ para designar a energia de repouso e $m$ para designar a massa de repouso.

O resultado de Einstein originalmente derivado em (1905b) é chamado às vezes (por exemplo, Baierlein, 2007) de a versão "incremental" de (3) , que agora podemos escrever, usando as convenções que acabamos de adotar para os símbolos $E$ e $m$, como:

$$
\Delta E=\Delta m c^{2}
$$

onde $\Delta E$ designa uma mudança na energia de $S$ e $\Delta m$ designa uma mudança na massa de $S$. Se reescrevermos (4), dividindo ambos os lados por $c^{2}$, podemos ver que (4) também diz que se a energia de $S$ muda por uma quantidade $\Delta E$, então sua massa muda por uma quantidade $\Delta m=$ $\Delta E / c^{2}$

\subsection{Exemplos de $E=m c^{2}$ em funcionamento}

Para ilustrar as implicações físicas de (4), físicos tendem a usar dois tipos principais de exemplos: (i) exemplos que examinam a massa e energia de um único corpo como um todo não-analisado, e (ii) exemplos que examinam a massa e energia de uma coleção de objetos, especialmente objetos atômicos e sub-atômicos envolvidos em colisões. Esta última classe de exemplos é particularmente útil para a compreensão da equivalência massa-energia porque lidam com as mudanças em energias e massas que são detectáveis. Além disso, tais exemplos podem enfatizar a importância de se considerar toda a energia de um sistema físico, incluindo a energia potencial dos seus constituintes, no cálculo da massa (e energia) total desse sistema.

Começamos discutindo a massa e energia de um corpo único (na Seção 1.2.1). Como uma conexão para a nossa discussão de colisões entre objetos sub-atômicos (na Seção 1.2.3), primeiro discutiremos a massa e energia de um gás ideal (na Seção 1.2.2). Em cada caso, identificaremos cuidadosamente o sistema físico em consideração, porque uma falha nesse passo pode levar a confusão interpretativa.

\subsubsection{Massa e energia de um único corpo}

Vamos primeiro supor que o nosso sistema físico $S$ consiste de $1 \mathrm{~kg}$ de barra de ouro que absorve energia térmica suficiente para que sua temperatura aumente em $10^{\circ} \mathrm{C}$. Como resultado da absorção de energia de calor, a massa inercial da barra de ouro aumenta em 1,4 $\times$ $10^{-14} \mathrm{~kg}$. O aumento da massa é pequena, por causa do fator $c^{2}$ que divide $\Delta E$. Similarmente, 
se a barra de ouro irradia calor de modo que a sua temperatura diminui em $10^{\circ} \mathrm{C}$, então sua massa inercial irá diminuir simultaneamente por $1,4 \times 10^{-14} \mathrm{~kg}$.

Neste exemplo, a nova exigência feita pela relatividade restrita é de que a massa inercial de um sistema físico muda quando o sistema absorve ou emite energia. Tal mudança não ocorre de acordo com a física pré-relativística. Na física pré-relativística, a massa inercial da barra de ouro, isto é, a tendência da barra para resistir às mudanças de velocidade é a mesma em todas as temperaturas.

Note que neste exemplo tratamos as fronteiras da barra de ouro como as fronteiras do nosso sistema físico. Porém, como um sistema físico, a barra de ouro não está isolada, porque interage com seu meio ambiente. Colocando de forma ligeiramente diferente, quando a barra de ouro absorve (ou emite) energia, sua massa inercial aumenta (ou diminui), porque há um fluxo líquido de energia para dentro (ou para fora) da barra de ouro.

Pode-se, é claro, perguntar o que acontece "dentro" da barra de ouro para que haja uma mudança em sua massa inercial, à medida que se altera seu conteúdo de energia Para responder a esta pergunta, é preciso examinar a barra de ouro no nível atômico. Claramente, podemos realizar tal análise. Uma vez que $E=m c^{2}$ rege o comportamento de todos os sistemas físicos, podemos usá-lo para explorar sistemas físicos mais complexos, como o sistema de átomos que constitui a barra de ouro.

No entanto, para simplificar, vamos considerar as mudanças análogas à massa e energia de um gás ideal. Um gás ideal constitui um sistema físico mais simples do que a barra de ouro, porque na análise do primeiro não precisamos levar em conta a energia potencial dos constituintes que compõem o sistema físico (que é algo que não podemos ignorar, se analisarmos a barra de ouro a nível atômico).

\subsubsection{Massa e energia de um gás ideal}

Suponhamos agora que o nosso sistema físico $S$ é um gás ideal, ou seja, uma coleção de partículas pontuais idealizadas que se movem sem sofrer a ação de força alguma e que colidem umas com as outras dentro de um recipiente de massa desprezível. Para um tal sistema $S$, a massa inercial de $S$ é a soma (i) das massas de repouso de todas as partículas individuais que compõem o gás e (ii) da soma das energias cinéticas de todas as partículas divididas por $c^{2}$. Não há outro componente que contribua para a massa inercial de $S$, uma vez que as partículas não têm qualquer energia potencial. Por conseguinte, se a energia cinética 
média das moléculas de gás diminui, como no caso de um resfriamento do gás, então a massa inercial da amostra de gás diminui. Em outras palavras, a tendência de todo o recipiente de gás para resistir a alterações da velocidade irá diminuir à medida que a temperatura do gás diminui. Mais uma vez, esta é uma nova previsão da relatividade restrita que está ausente na física pré-relativística.

A equação de Einstein também diz, é claro, que se houver uma alteração na massa inercial de $S$, então existe ao mesmo tempo uma mudança na energia de repouso de $S$. Assim, se removermos uma molécula da amostra de gás, a energia de repouso da amostra de gás irá diminuir por uma quantidade igual à soma da energia cinética da molécula $e$ da massa da molécula vezes $c^{2}$. Podemos modificar o nosso exemplo para torná-lo um pouco mais expressivo se considerarmos que a amostra de gás esteja a uma temperatura de zero absoluto, isto é, se considerarmos a amostra de gás quando todas as suas moléculas estão em um estado de repouso relativo. Neste caso, a energia de repouso de $S$ é simplesmente a soma das massas das moléculas vezes $c^{2}$. Suponhamos, para simplificar, que existem $n$ moléculas cada uma com massa de repouso $m$. A energia de repouso de $S$ é, então, simplesmente $E=n \cdot m c^{2}$. Se removermos uma das moléculas do gás, então a energia de repouso diminui por valor $\Delta E=$ $m c^{2}$ e a nova energia de repouso de $S$ torna-se $E^{\prime}=(n-1) m c^{2}$.

Note-se que, durante toda nossa discussão do gás ideal, implicitamente supusemos que as fronteiras do sistema $S$ são as paredes do recipiente. Quando vimos que a massa de $S$ diminuía, era porque $S$ irradiava energia na forma de calor. Note bem que, neste exemplo, $S$ não é um sistema isolado, porque existe um fluxo líquido de energia para fora de $S$ e para seu ambiente circundante. Similarmente, se a amostra de gás absorvesse energia, digamos por absorção de radiação eletromagnética através de suas fronteiras, então sua massa inercial aumentaria.

Consideremos agora duas tais amostras de gás $S_{1}$ e $S_{2}$ que estão a uma certa distância entre si em um vácuo. Suponhamos ainda que toda a energia $\Delta E$ emitida por $S_{1}$ é absorvida por $S_{2}$. Nesta configuração, a massa $M_{1}$ de $S_{1}$ irá diminuir por uma quantidade $\Delta E / c^{2}$ Como $S_{2}$ absorve a quantidade de energia $\Delta E$, sua massa aumenta por uma quantidade igual $\Delta E / c^{2 .}$ Há um sentido, então, no qual pode ser dito que a radiação que "transportou" a energia $\Delta E$ de $S_{1}$ a $S_{2}$ teve o efeito de transferir uma parte da massa inercial de $S_{1}$ a $S_{2}$, ou como Einstein colocou: "Se a teoria concorda com os fatos, então a radiação transmite inércia entre o corpo emissor e o absorvedor" (1905b, p. 174). 
Vamos agora considerar uma configuração física diferente. Suponha que nossa amostra original de gás $S$ está dentro de um recipiente maior $S^{\prime}$. Vamos ainda supor que as paredes interiores de $S^{\prime}$ são superfícies refletoras perfeitas e que as paredes de $S$ são espelhos unidirecionais com refletores externos perfeitos. O que acontece com a massa inercial de $S^{\prime}$, quando o gás esfria em $S$ ?

À medida que o gás em $S$ esfria, uma parte da energia cinética das suas moléculas é transformada em calor, e esta energia térmica liberada por $S$ escapa para o interior de $S^{\prime}$. No entanto, como estamos supondo que as paredes interiores de $S^{\prime}$ e as paredes exteriores de $S$ são completamente refletoras, a energia térmica não escapa de $S^{\prime}$. Assim, enquanto o gás esfria a massa inercial de $S^{\prime}$ permanece constante. A mudança que ocorreu dentro de $S^{\prime}$ é que uma parte da energia cinética das moléculas dentro de $S$ tornou-se a energia térmica presa fora de $S$ e no interior de $S^{\prime}$. No entanto, isso não tem nenhum efeito sobre a massa de $S^{\prime}$, que é simplesmente a soma das massas das moléculas em $S$ mais a energia total contida dentro de $S^{\prime}$ dividido por $c^{2}$. Não importa como a energia é distribuída dentro de $S^{\prime}$. Para colocar de forma ligeiramente diferente, já que estamos tratando $S^{\prime}$ como um sistema isolado, a sua massa inercial deve permanecer constante (mesmo de acordo com a relatividade restrita).

\subsubsection{Massa e energia em colisões de partículas pontuais}

Talvez os exemplos mais comuns utilizados para ilustrar a equação de Einstein digam respeito às colisões entre objetos subatômicos. Para os nossos propósitos, é seguro tratar os objetos atômicos e subatômicos como partículas envolvidas em colisões onde o número total de partículas pode ou não ser conservado.

O bombardeamento de um núcleo de lítio por prótons é um exemplo de importância histórica e útil para a discussão da equivalência massa-energia em colisões onde o número de partículas é conservado. Cockcroft \& Walton (1932) foram os primeiros a observar a emissão de duas partículas alfa $(\alpha)$, quando um próton $p$ colide com um núcleo ${ }^{7} \mathrm{Li}$. A reação é rotineiramente simbolizada como se segue:

$$
p+{ }^{7} \mathrm{Li} \rightarrow \alpha+\alpha
$$

Que o número de partículas é conservado na reação (ㅁ) torna-se claro quando reconhecemos que o núcleo ${ }^{7} \mathrm{Li}$ é constituído por três prótons e quatro nêutrons e que cada partícula $\alpha$ consiste de dois prótons e dois nêutrons. 
Na reação (5), a soma das massas de repouso dos reagentes (o próton e o núcleo ${ }^{7} \mathrm{Li}$ ) é maior do que a soma das massas de repouso dos produtos (as duas partículas $\alpha$ ). Em compensação, a energia cinética total dos reagentes é menor do que a energia cinética total dos produtos. O experimento de Cockcroft \& Walton é rotineiramente interpretado como uma demonstração de que a diferença das massas de repouso dos produtos e reagentes (vezes $c^{2}$ ) é igual à diferença nas energias cinéticas dos produtos e reagentes (mas veja a Seção 4 para discussão adicional sobre esta experiência como uma confirmação de equivalência massaenergia).

Descrições de colisões entre partículas subatômicas como as que demos acima sugerem que é preciso admitir que a massa é convertida em energia. No entanto, talvez influenciado pela discussão muito conhecido de equivalência massa-energia de Bondi \& Spurgin (1987), os físicos agora explicam tais reações não como casos de massa que é convertida em energia, mas apenas como casos onde a energia mudou de forma. Tipicamente, nestes tipos de reações, a energia potencial, que "contribui" para a massa de repouso de um (ou possivelmente mais) dos reagentes, é transformada de uma forma não-controversa em energia cinética dos produtos. Como Baierlein (2007, p. 322) explica, no caso do bombardeio de ${ }^{7} \mathrm{Li}$ com prótons e sua subsequente decomposição em duas partículas $\alpha$, o aparente "excesso" de energia cinética das partículas $\alpha$ não "aparece" simplesmente do nada. Em vez disso, aquela energia estava lá o tempo todo como energia potencial e energia cinética dos núcleons (prótons e nêutrons). Em outras palavras, pode-se explicar a alteração na massa e energia na reação (ㅁ) dizendo (i) que as energias potenciais e cinéticas dos núcleons que compõem o núcleo ${ }^{7} \mathrm{Li}$ contribuem para a sua massa de repouso e (ii) que a grande quantidade de energia das partículas $\alpha$ não foi "criada" na reação, ou "convertida" a partir de massa, mas foi simplesmente transformada a partir das várias formas de energia que os núcleons possuíam. Claro que, precisamente o que significa dizer que a energia dos núcleons neste exemplo pode "contribuir" para a massa do núcleo permanece pouco claro nesta fase. Discutimos essa questão na Seção 2 .

As colisões entre partículas subatômicas em que o número de partículas não é conservado não são tão facilmente explicadas como meramente envolvendo o rearranjo de partículas e redistribuição de energia. O exemplo mais extremo deste tipo, e que é frequentemente utilizado na literatura física, é a aniquilação de pares. Por conseguinte, vamos considerar uma colisão entre um elétron $e^{-}$e um pósitron $e^{+}$, que produz dois fótons $(2 \gamma)$. Simbolicamente, a reação é escrita como se segue: 


$$
e^{-}+e^{+} \rightarrow \gamma+\gamma
$$

De acordo com o atualmente aceito Modelo Padrão da física de partículas, elétrons e fótons são ambos "partículas fundamentais", pelo qual os físicos querem dizer que essas partículas não têm estrutura, ou seja, estas partículas não são compostas por outras partículas menores. Além disso, os fótons que são os produtos na reação (ㅁ) têm massa de repouso nula. Assim, na reação (6), as massas de repouso do elétron e do pósitron incidentes parecem "desaparecer", e uma quantidade equivalente de energia "aparece" como a energia dos fótons que saem. Claro que a famosa equação de Einstein faz todas as previsões corretas quanto às massas e energias relevantes envolvidas na reação (ㅁ). Assim, por exemplo, a energia total dos dois fótons é igual à soma das energias cinéticas do elétron e do pósitron mais a soma das massas de repouso do elétron e do pósitron divididas por $c^{2}$.

Finalmente, embora a massa e a energia parecem "desaparecer" e "aparecer", respectivamente, quando consideramos os constituintes individuais do sistema físico que contém o par incidente elétron-pósitron $e$ os fótons de saída, a massa e energia de todo $o$ sistema permanecem a mesma por toda a interação. Antes da colisão, a massa de repouso do sistema é simplesmente a soma das massas de repouso do elétron e pósitron mais o equivalente de massa da energia cinética total das partículas. Por conseguinte, todo o sistema (se desenharmos a fronteira do sistema em torno dos reagentes e produtos - que é, naturalmente, uma fronteira espacial e temporal) tem uma massa de repouso não-nula antes da colisão. No entanto, após a colisão, o sistema, que agora é constituído por dois fótons que se deslocam em direções não paralelas, também tem uma massa de repouso diferente de zero (ver, por exemplo, Taylor \& Wheeler, 1992, p. 232). Nós discutimos como este tipo de reação de aniquilação está relacionado com interpretações de $E=m c^{2} \operatorname{logo}$ abaixo na Seção 2 .

\section{Interpretações filosóficas de $\mathbf{E}=\mathbf{m c}^{2}$}

Existem três principais questões filosóficas a respeito da interpretação de $E=m c^{2,}$ que têm ocupado filósofos e físicos:

1. Serão massa e energia a mesma propriedade dos sistemas físicos, e é isso que se quer dizer ao afirmar que elas são "equivalentes”? 
2. A massa é "convertida" em energia em algumas interações físicas, e em caso afirmativo, qual é o sentido relevante de "conversão"?

3. Será que $E=m c^{2}$ tem consequências ontológicas, e em caso afirmativo, quais são elas?

Interpretações da equivalência massa-energia podem ser organizadas de acordo com como responde-se às perguntas (1) e (2) acima. Como veremos (na Seção 2.5), as interpretações que respondem à pergunta (3) afirmativamente assumem que a resposta à pergunta (1) é sim.

A única combinação de respostas às perguntas (1) e (2) que é inconsistente é dizer que a massa e energia são a mesma propriedade dos sistemas físicos, mas que a conversão de massa em energia (ou vice-versa) é um genuíno processo físico. Todas as outras três combinações de respostas às perguntas (1) e (2) são opções viáveis e têm sido defendidas, em algum momento ou outro, por físicos ou filósofos como indicado pelos exemplos dados na Tabela 1.

Tabela 1: Interpretações da equivalência massa-energia

\begin{tabular}{|l|c|c|}
\hline & Conversão & Não Conversão \\
\hline Mesma propriedade & X & Torretti (1996), Eddington (1929) \\
\hline Propriedades diferentes & $\begin{array}{c}\text { Rindler (1977) } \\
\text { (conversão é possível) }\end{array}$ & Bondi \& Spurgin (1987) \\
\hline
\end{tabular}

Nesta seção, vamos descrever os méritos e deméritos de cada uma das interpretações da Tabela 1. Além dessas interpretações, vamos discutir também outros dois tipos de interpretações da equivalência massa-energia que não se encaixam perfeitamente na Tabela 1. Primeiro, vamos discutir a recente interpretação de Lange $(2001,2002)$ que afirma que somente a massa é uma propriedade real de sistemas físicos e que nós convertemos massa em energia, quando mudamos o nível em que analisamos os sistemas físicos. Em segundo lugar, vamos discutir duas interpretações (uma de Einstein \& Infeld, 1938 e outra de Zahar, 1989), que chamaremos de interpretações ontológicas, que tentam responder à pergunta (3) afirmativamente. No entanto, começamos esta seção abordando o que era anteriormente um equívoco bastante comum concernente à equivalência massa-energia. 
Queremos salientar que a forma geral na qual categorizamos e explicamos as interpretações da equivalência massa-energia nesta seção apareceram pela primeira vez em Flores (2005).

\subsection{Equívocos sobre $E=m c^{2}$}

Embora seja muito menos comum hoje em dia, ainda se ouve por vezes que a equação de Einstein implica que a matéria pode ser convertida em energia. Estritamente falando, este constitui um elementar erro de categoria. Na física relativística, como na física clássica, massa e energia são tidos como propriedades de sistemas físicos ou propriedades dos constituintes de sistemas físicos. Se alguém deseja falar sobre as coisas físicas que são portadoras de tais propriedades, então geralmente fala sobre "matéria" ou "campos". A distinção entre "matéria" e "campos" na física moderna é em si bastante sutil em grande parte por causa da equivalência entre massa e energia. Entretanto, podemos afirmar que qualquer sentido de "conversão" que parece convincente entre massa e energia, terá de ser uma "conversão" entre massa e energia, e não entre matéria e energia. Finalmente, nossa observação vale mesmo nas chamadas reações de "aniquilação" onde toda a massa das partículas incidentes parece “desaparecer" (ver, por exemplo, Baierlein, 2007, p. 323). Claro, a terminologia mais antiga de "matéria" e "anti-matéria" não ajuda na nossa compreensão filosófica da equivalência massa-energia, e tem talvez parte da culpa pelos equívocos que cercam $E=m c^{2}$

\subsection{Interpretações de mesma-propriedade de $E=m c^{2}$}

A primeira interpretação que vamos considerar responde "sim" à primeira pergunta interpretativa colocada acima: massa e energia são a mesma propriedade dos sistemas físicos. Consequentemente, não há nenhum sentido em que uma das propriedades é fisicamente convertida em outra.

Filósofos como Torretti (1996) e físicos, tais como Eddington (1929) adotaram a interpretação de mesma-propriedade. Por exemplo, Eddington afirma que "parece muito provável que massa e energia são duas maneiras de medir o que é essencialmente a mesma coisa, no mesmo sentido que paralaxe e distância de uma estrela são dois modos de exprimir a mesma propriedade de localização" (1929, p. 146). Segundo Eddington, a distinção entre massa e energia é artificial. Nós tratamos massa e energia como diferentes propriedades dos sistemas físicos porque rotineiramente as medimos usando diferentes unidades. No entanto, 
pode-se medir a massa e a energia usando as mesmas unidades, escolhendo unidades para as quais $c=1$, isto é, unidades em que as distâncias são medidas em unidades de tempo (por exemplo, anos-luz). Uma vez que fazemos isso, sustenta Eddington, a distinção entre massa e energia desaparece.

Como Eddington, Torretti aponta que massa e energia parecem ser diferentes propriedades, porque são medidas em unidades diferentes. Falando contra a visão da Bunge (1967), segundo a qual a sua equivalência numérica não implica que massa e energia "são a mesma coisa", Torretti explica:

Se uma geladeira da cozinha pode extrair a massa de uma determinada jarra de água e transferi-la por radiação térmica ou convecção para a parede da cozinha por trás dela, uma distinção metafísica incisiva entre a massa e a energia da matéria parece improvável (1996, p. 307, nota 13).

Para Torretti, a própria existência de processos físicos em que a emissão de energia por um objeto está correlacionada com a diminuição da massa do objeto, de acordo com a equação de Einstein, vai fortemente contra a visão de que massa e energia são, de alguma forma, distintas propriedades de sistemas físicos. Torretti continua:

Claro que, se os comprimentos e tempos são medidos com unidades diferentes e independentes, a 'massa' [...] difere conceitualmente da 'energia' [...]. Mas essa diferença pode ser entendida como uma consequência do conveniente, mas enganoso, ato da mente pelo qual abstraímos tempo e espaço da natureza (1996, p. 307, nota 13).

Assim, esta nota de rodapé em seu magistral Relatividade e Geometria sugere que, para Torretti, somos levados à utilização de unidades diferentes para a massa e a energia meramente pela forma como percebemos o espaço e o tempo. Como vimos, pode-se utilizar as mesmas unidades de massa e energia ao adotar-se a convenção que o próprio Torretti utiliza ao selecionar unidades em que $c=1$ (pp. 88-89). No entanto, pode ser útil lembrar que apenas usando as mesmas unidades de intervalos temporais e espaciais não implica que o espaço e o tempo são tratados "em pé de igualdade" na relatividade restrita; eles não são, como é evidente a partir da assinatura da métrica de Minkwoski.

O principal mérito da visão de Torretti é que ela leva muito a sério a unificação do espaço e do tempo feita pela teoria da relatividade especial e tão famosamente anunciada nas primeiras linhas de Minkowski (1908). Também é consistente com a forma como massa e energia são tratadas na relatividade geral. 
Interpretações como as de Torretti e de Eddington não tiram outras conclusões ontológicas a partir da equivalência massa-energia. Por exemplo, nem Eddington nem Torretti fazem qualquer afirmação explícita sobre se as propriedades são melhor entendidas como universais, ou se deve-se ser um realista sobre tais propriedades. Por fim, ao afirmarem que massa e energia são a mesma coisa, esses pensadores estão sugerindo que a denotação dos termos "massa" e "energia" é o mesmo, embora reconheçam que a conotação destes termos é claramente diferente.

\subsection{Interpretações de diferentes-propriedades de $E=m c^{2}$}

Como já apresentado na Tabela 1, as interpretações da equivalência de massa-energia que sustentam que massa e energia são propriedades diferentes discordam sobre se existe algum processo físico, através do qual a massa é convertida em energia (ou vice-versa). Embora a recente interpretação de Lange $(2001,2002)$ parece cair superficialmente nessa categoria, dado que ele certamente trata massa e energia como propriedades diferentes, ele difere de outros nesta categoria, porque Lange explicitamente argumenta que somente a massa é uma propriedade real de sistemas físicos. Sendo assim, vamos discutir a interpretação de Lange separadamente (na Seção 2.3.3).

Começaremos com uma discussão da interpretação de Bondi \& Spurgin (na Seção 2.3.1). Eles sustentam que massa e energia são propriedades distintas e que não há tal coisa como a conversão de massa e energia. Discutiremos então a interpretação de Rindler (na Seção 2.3.2). Ele afirma que massa e energia são propriedades diferentes, mas que conversões genuínas de massa e energia são pelo menos permitidas pela equivalência massa-energia.

\subsubsection{A interpretação de diferentes-propriedades e não-conversão de Bondi \& Spurgin}

A interpretação de Bondi \& Spurgin (1987), de equivalência massa-energia tem sido influente, especialmente entre os físicos envolvidos com a área educacional. Em um artigo onde eles se queixaram sobre como os alunos muitas vezes equivocam-se sobre a famosa equação de Einstein, Bondi e Spurgin argumentaram que a equação de Einstein implica que massa e energia são a mesma propriedade, tanto quanto a equação $m=\rho V$ (onde $m$ é a massa, $V$ é o volume e $\rho$ é a densidade) implica que a massa e volume são os mesmos. Tal como no caso de massa e volume, Bondi \& Spurgin argumentam que massa e energia têm dimensões diferentes. Em última análise, isso reduz a uma discordância com filósofos como Torretti que 
argumentariam que o tempo, como uma dimensão, não é diferente de qualquer uma das dimensões espaciais. Note bem que isto não é uma questão sobre as unidades que usamos para medir a massa (ou energia).

Todos concordam que, de acordo com a relatividade restrita, podem-se medir intervalos espaciais em unidades de tempo. Podemos fazer isso por causa do postulado da relatividade restrita que diz que a velocidade da luz tem o mesmo valor em todos os referenciais inerciais. Se executarmos o que equivale a uma substituição de variáveis e levarmos nossas dimensões espaciais para serem $x_{N}{ }^{*}=x_{n} / c$, onde $c$ é a velocidade da luz e $n=$ $1,2,3$, podemos escolher unidades em que $c=1$.

No entanto, pode-se usar consistentemente unidades nas quais $c=1$ e sustentar que há, mesmo assim, uma distinção fundamental entre o espaço e o tempo como dimensões. Em tal visão, que é a visão que Bondi \& Spurgin parecem implicitamente defender, enquanto o tempo é distinto de qualquer dada dimensão espacial, o fato contingente de que $c$ tem o mesmo valor em todos os referenciais inerciais nos permite efetuar a relevante substituição de variáveis. No entanto, não se segue daí que devemos tratar o tempo em pé de igualdade com qualquer dimensão espacial, ou que devemos tratar o intervalo espaço-temporal como mais fundamental (do modo que Torretti faz).

Em seu influente artigo, Bondi \& Spurgin examinam uma variedade de casos de supostas conversões de massa e energia. Em cada caso, eles mostram que a suposta conversão de massa e energia é melhor entendida apenas como uma transformação da energia. Em geral, Bondi \& Spurgin argumentam, sempre que encontramos uma suposta conversão de massa e energia, podemos explicar o que está acontecendo olhando para os componentes do sistema físico na reação e examinando como a energia é distribuída entre os componentes antes e após a reação ocorrer.

Como vimos acima, na interpretação mínima de $E=m c^{2}$ (Seção 1.2.3), explicações sobre as supostas "conversões", nas linhas sugeridas por Bondi \& Spurgin, são agora comuns na literatura física. Essas explicações têm o mérito de enfatizar que, em muitos casos, os mistérios da equivalência massa-energia não dizem respeito a uma propriedade física magicamente sendo transfigurada em outra. No entanto, a interpretação de Bondi-Spurgin de equivalência massa-energia tem o demérito de não conseguir resolver reações como a reação de aniquilação elétron-pósitron (므). Em tais reações, não só é o número de partículas não conservado, mas todas as partículas envolvidas são, por hipótese, totalidades indivisíveis. Assim, a energia liberada em reações deste tipo não pode ser explicada como resultado de 
uma transformação da energia que foi originalmente possuída pelos constituintes das partículas que reagem. Claro, Bondi \& Spurgin podem estar simplesmente esperando que a física revelará que as partículas, como elétrons e pósitrons não são totalidades indivisíveis no fim das contas. $\mathrm{Na}$ verdade, eles podem até usar as reações de aniquilação combinadas com a sua interpretação da equivalência massa-energia para argumentar que não pode ser o caso de que essas partículas são indivisíveis. Assim, assistimos aqui explicitamente o quão intimamente relacionadas as interpretações sobre equivalência massa-energia podem estar de pontos de vista sobre a natureza da matéria.

O segundo demérito da interpretação de Bondi-Spurgin, que é compartilhado com todas as demais interpretações da equivalência massa-energia que sustentam que massa e energia são propriedades diferentes, é que ela permanece em silêncio sobre uma característica central de sistemas físicos, que ela usa para explicar aparentes conversões de massa e energia. A fim de explicar as supostas conversões nas linhas sugeridas por Bondi-Spurgin, deve-se fazer a familiar suposição de que a energia dos constituintes de um sistema, seja energia potencial ou energia cinética, "contribui" para a massa de repouso do sistema. Assim, por exemplo, no bombardeamento e subsequente decomposição de ${ }^{7} \mathrm{Li}$, isto é, na reação (ㅁ) , Bondi \& Spurgin devem explicar a massa de repouso do ${ }^{7} \mathrm{Li}$ da forma familiar, como surgindo de ambas a soma das massas de repouso dos núcleons e as massas equivalentes de suas energias. No entanto, a interpretação de Bondi-Spurgin não oferece qualquer explicação sobre porque as energias dos constituintes de um sistema físico, seja energia potencial ou energia cinética, manifestam-se como parte da massa inercial do sistema como um todo.

Como veremos, a interpretação de Rindler da equivalência massa-energia tenta resolver o primeiro demérito da interpretação de Bondi-Spurgin, enquanto a interpretação de Lange traz para o primeiro plano que a energia dos constituintes de um sistema físico "contribui” para a massa inercial do sistema.

\subsubsection{A interpretação de diferentes-propriedades e conversão de Rindler}

A interpretação de Rindler da equivalência massa-energia é uma versão ligeiramente modificada, todavia de maneira importante, da interpretação de Bondi-Spurgin. Rindler (por exemplo, em 1977), concorda que existem muitas supostas conversões que são melhor entendidas como meras transformações de um tipo de energia para um tipo diferente de energia. Assim, Rindler também adota a interpretação mínima da equivalência de massaenergia, por exemplo, no bombardeamento e subsequente decomposição de ${ }^{7} \mathrm{Li}$. 
No entanto, para Rindler, não há nada dentro da relatividade restrita em si que exclui a possibilidade de que existam partículas fundamentais, sem estrutura (ou seja, partículas que são "atômicas" no sentido filosófico do termo). Se tais partículas existem, é possível, de acordo com a equação de Einstein, que alguma parte ou toda a massa de tais partículas "desapareça" e uma quantidade equivalente de energia "apareça" dentro do sistema físico relevante. Assim, Rindler parece estar sugerindo que devemos limitar a nossa interpretação da equivalência massa-energia ao que podemos deduzir da relatividade restrita. Assim, devemos sustentar que a equação de Einstein, pelo menos, permite conversões genuínas de massa em energia, no sentido de que pode haver casos em que uma certa quantidade de massa inercial "desaparece" de dentro de um sistema físico e uma quantidade correspondente de energia “aparece". Além disso, em tais casos, não podemos explicar a reação como meramente envolvendo uma transformação de um tipo de energia para outra.

O mérito da interpretação de Rindler é que limita a interpretação da equação de Einstein para o que pode-se validamente inferir a partir dos postulados da relatividade restrita. Ao contrário da interpretação proposta por Bondi \& Spurgin, a interpretação de Rindler não faz suposições sobre a constituição da matéria.

\subsubsection{A interpretação de uma-propriedade e não-conversão de Lange}

Lange (2001, 2002) sugeriu recentemente uma interpretação especial de equivalência massa-energia. Lange começa sua interpretação argumentando que a massa de repouso é a única propriedade real de sistemas físicos. Esta afirmação por si só sugere que não pode haver tal coisa como um processo físico pelo qual a massa é convertida em energia, pois, como Lange pergunta, "em que sentido pode a massa ser convertida em energia quando a massa e energia não estão em igualdade em termos de sua realidade?” (2002, p. 227, ênfase no original). Lange, em seguida, continua a argumentar que uma análise cuidadosa de supostas conversões de massa-energia revela que não há nenhum processo físico pelo qual a massa é convertida em energia. Em vez disso, argumenta Lange, a aparente conversão de massa em energia (ou vice-versa) é uma ilusão que surge quando mudamos o nosso nível de análise ao examinarmos um sistema físico.

Lange parece usar um argumento familiar, que parte da invariância de Lorentz de certas grandezas físicas e conclui por sua "realidade". Para Lange, se uma grandeza física não é uma invariante de Lorentz, então não é real no sentido de que ela não representa "os fatos objetivos, em que todos os referenciais inerciais concordam" (2002, p. 209). Assim, Lange 
utiliza a invariância de Lorentz como uma condição necessária para a realidade de uma grandeza física. No entanto, em vários outros lugares, por exemplo, quando Lange defende a realidade do intervalo de Minkowski (2002, p. 219) ou quando ele defende a realidade da massa de repouso (2002, p. 223), Lange utiliza implicitamente a invariância de Lorentz como uma condição suficiente para a realidade de uma grandeza física. No entanto, se Lange adota a invariância de Lorentz tanto como uma condição necessária quanto suficiente para a realidade de uma grandeza física, então ele está comprometido com a visão que a energia de repouso é real pelos mesmos motivos que ele está comprometido com a visão que a massa de repouso é real. Assim, a sugestão original de Lange de que não pode haver processo físico de conversão entre massa e energia, porque eles têm estatuto ontológico diferente, parece desafiado.

Acontece que a posição geral de Lange não está seriamente desafiada pelo estatuto ontológico da energia de repouso. Lange poderia facilmente conceder que essa energia de repouso é uma propriedade real de sistemas físicos e ainda argumentar (i) que não há tal coisa como um processo físico de conversão entre massa e energia e (ii) que supostas conversões resultam da mudança de níveis de análise quando examinamos um sistema físico. São suas observações relativamente a (ii) que nos forçam a enfrentar uma vez mais a questão de porque a energia dos constituintes de um sistema físico manifesta-se como a massa do sistema. A interpretação de Lange, infelizmente, não nos leva mais próximo de responder a essa pergunta, embora, como vamos sugerir a seguir, nenhuma interpretação da equivalência massa-energia pode fazer isso (ver Seção 3.2).

Um dos principais exemplos que Lange utiliza para apresentar sua interpretação da equivalência massa-energia é o aquecimento de um gás ideal, que já consideramos acima (ver Seção 1.2.2). Ele também considera exemplos que envolvem reações entre partículas subatômicas que, para nossos propósitos, são muito semelhantes nos aspectos relevantes ao exemplo que discutimos sobre o bombardeio e subsequente decomposição de um núcleo de ${ }^{7}$ Li. Em ambos os casos, Lange essencialmente adota a interpretação mínima acerca da qual discutimos acima. No caso do gás ideal, como já vimos, quando a amostra de gás é aquecida e sua massa inercial ao mesmo tempo aumenta, este aumento na massa de repouso não é resultado de o gás de algum modo estar subitamente (ou gradualmente) composto de moléculas que são elas próprias mais massivas. Também não é o resultado de o gás de repente (ou gradualmente) conter mais moléculas. Em vez disso, o aumento da energia cinética das moléculas do gás "contribui” para o aumento da massa inercial da amostra de gás. Lange resume essa característica do aumento da massa inercial da amostra de gás, dizendo: 
“[...] acabamos de ver que esta 'conversão' de energia em massa não é um processo físico real de modo algum. Nós 'convertemos' energia em massa simplesmente por uma mudança de nossa perspectiva sobre o gás: a passagem de inicialmente tratá-lo como muitos corpos para tratá-lo como um corpo único" [ênfase no original] (2002, p. 236)

Infelizmente, a caracterização de Lange ameaça deixar os leitores com a impressão de que se "nós" não tívessemos mudado a nossa perspectiva na análise do gás, nenhuma alteração da massa inercial da amostra de gás teria se seguido. Claro que, é improvável que Lange queira dizer isso. Certamente, Lange concordaria que, mesmo se os seres humanos não estão por perto para analisar uma amostra de gás, a amostra de gás passará a responder, em qualquer interação física, de forma diferente como um todo depois de ter absorvido alguma energia, precisamente porque a sua massa inercial terá aumentado.

Os méritos da visão de Lange concernentes à "conversão" de massa e energia são essencialmente os mesmos que os méritos tanto da interpretação de Bondi-Spurgin quanto da interpretação de Rindler. Em todos os casos, essas interpretações concordam com a interpretação mínima de $E=m c^{2}$, de que há casos importantes dos quais temos agora aprendido o suficiente para afirmar com segurança que supostas "conversões" de massa e energia são apenas casos onde a energia de uma espécie se transforma em energia de outro tipo. Para além da questão comparativamente menor sobre a "realidade" da energia de repouso, o demérito principal da visão de Lange é a de que ele poderia potencialmente enganar leitores desavisados.

\subsection{Interpretações de $E=m c^{2}$ e hipóteses sobre a natureza da matéria}

A relação entre a equivalência massa-energia e hipóteses sobre a natureza da matéria se dá de duas maneiras. Primeiro, como já sugerimos implicitamente, algumas das interpretações da equivalência massa-energia parecem supor certas características da matéria. Segundo, alguns filósofos e físicos, especialmente Einstein \& Infeld (1938) e Zahar (1989), argumentaram que a equivalência massa-energia tem consequências sobre a natureza da matéria. Nesta seção, vamos discutir a primeira dessas duas relações entre $E=m c^{2}$ e hipóteses sobre a natureza da matéria. Discute-se a segunda relação na próxima seção (Seção 2.5).

Para explicar como algumas interpretações da equivalência massa-energia baseiam-se em suposições sobre a natureza da matéria, precisamos primeiro reconhecer, como vários 
autores já apontaram, por exemplo, Rindler (1977), Stachel \& Torretti (1982), e Feigenbaum \& Mermin (1988), que a relação que realmente deriva da relatividade restrita é:

$$
E=(m-q) c^{2}+K
$$

onde $K$ é meramente um fator aditivo que fixa o ponto zero de energia e é convencionalmente definido como zero, e $q$ também é rotineiramente definido como sendo zero. No entanto, ao contrário da convenção para definir $K$ como sendo zero, estipular que $q=0$ envolve uma hipótese sobre a natureza da matéria, porque exclui a possibilidade de que existe matéria que tem massa, mas que é tal que um pouco de sua massa nunca pode ser "convertido" em energia.

A interpretação de mesma-propriedade da equivalência massa-energia repousa inequivocamente na suposição de que $q=0$. Massa e energia não podem ser a mesma propriedade se existe matéria que tem massa da qual uma parte não pode nunca, sob quaisquer condições, ser "convertida" em energia. No entanto, pode-se argumentar que, embora a interpretação de mesma-propriedade faz esta suposição, esta não é uma suposição injustificada. Atualmente, os físicos não têm qualquer evidência de que existe matéria para a qual $q$ não é igual a zero. No entanto, parece importante, do ponto de vista filosófico, reconhecer que a interpretação de mesma-propriedade depende não apenas daquilo que se pode derivar dos postulados da relatividade restrita, mas também de evidências de "fora" desta teoria.

As interpretações de $E=m c^{2}$ que sustentam que a massa e energia são propriedades distintas de sistemas físicos não necessitam, é claro, assumir que $q$ é diferente de zero. Tais interpretações podem simplesmente deixar que o valor de $q$ seja determinado empiricamente, pois, como vimos, tais interpretações argumentam que massa e energia sejam tratadas como propriedades distintas por razões diferentes. Mesmo assim, a interpretação Bondi-Spurgin parece adotar implicitamente uma hipótese sobre a natureza da matéria.

De acordo com Bondi \& Spurgin, todas as supostas conversões de massa e energia são casos em que um tipo de energia é transformado em outro tipo de energia. Isto, por sua vez, supõe que podemos, em todos os casos, compreender uma reação através da análise dos constituintes de sistemas físicos. Se nos concentrarmos em reações envolvendo partículas subatômicas, por exemplo, Bondi \& Spurgin parecem assumir que sempre podemos explicar tais reações, examinando a estrutura interna das partículas subatômicas. No entanto, se em 
algum momento encontrarmos boas evidências para apoiar a visão de que algumas partículas não têm estrutura interna, como agora parece ser o caso de elétrons, por exemplo, então temos que ou desistir da interpretação Bondi-Spurgin ou usar a própria interpretação para argumentar que tais partículas aparentemente sem estrutura, na verdade, contêm uma estrutura interna. Assim, parece que a interpretação Bondi-Spurgin supõe algo como a divisibilidade infinita da matéria, que é claramente uma hipótese que encontra-se "fora" da relatividade restrita.

\subsection{Interpretações ontológicas de $\mathrm{E}=m c^{2}$}

Einstein \& Infeld (1938) e Zahar (1989) argumentaram que $E=m c^{2}$ tem consequências ontológicas. Tanto a interpretação Einstein-Infeld quanto a interpretação de Zahar começam adotando a intepretação de mesma-propriedade de $E=m c^{2}$. Assim, de acordo com ambas as interpretações, massa e energia são as mesmas propriedades de sistemas físicos. Além disso, tanto a interpretação de Einstein-Infeld quanto a interpretação de Zahar usam uma distinção rudimentar entre "matéria" e "campos". De acordo com esta distinção um tanto ultrapassada, a física clássica inclui duas substâncias fundamentais: a matéria, que significa uma substância material ponderável, e campos, que significa campos físicos, como o campo eletromagnético. Para ambos, Einstein \& Infeld e Zahar, matéria e campos na física clássica são distinguidos pelas propriedades que carregam. A matéria tem tanto massa quanto energia, ao passo que os campos só têm energia. No entanto, uma vez que a equivalência massaenergia implica que massa e energia são, no final das contas, realmente a mesma propriedade física, dizem Einstein \& Infeld e Zahar, não se pode mais distinguir entre matéria e campos, pois ambos agora têm massa $e$ energia.

Embora tanto Einstein \& Infeld quanto Zahar usem o mesmo argumento básico, eles chegam a conclusões ligeiramente diferentes. Zahar argumenta que a equivalência massaenergia implica que a substância fundamental da física é uma espécie de "eu-não-sei-o-quê" que pode se manifestar como matéria ou campo. Einstein \& Infeld, por outro lado, em alguns lugares parecem argumentar que podemos inferir que a substância fundamental da física são campos. Em outros lugares, no entanto, parecem um pouco mais cautelosos e sugerem apenas que pode-se construir uma física somente com campos em sua ontologia.

Os deméritos de cada interpretação ontológica da equivalência massa-energia é que elas repousam sobre a interpretação de mesma-propriedade de $E=m c^{2}$. Como já discutimos anteriormente (ver Seção 2.4), enquanto pode-se adotar a interpretação de mesma-propriedade, 
para isso é preciso fazer suposições adicionais sobre a natureza da matéria. Além disso, a interpretação ontológica repousa sobre o que hoje parece uma distinção bastante rudimentar entre "matéria" e "campos". De fato, a equivalência massa-energia tem figurado proeminentemente na concepção dos físicos sobre a matéria em grande parte porque se abre a porta para uma descrição do que usualmente consideramos como matéria ponderável em termos de campos, uma vez que a energia do campo em um nível pode se manifestar como massa em um nível acima. No entanto, a inferência da equivalência massa-energia para a ontologia fundamental da física moderna parece muito mais sutil do que Einstein \& Infeld ou Zahar sugerem.

\section{História das derivações da equivalência massa-energia}

Einstein derivou pela primeira vez a equivalência massa-energia a partir dos princípios da relatividade restrita em um pequeno artigo intitulado "A inércia de um corpo depende de seu conteúdo de energia?" (1905b). Esta derivação, juntamente com outras que se seguiram logo após (por exemplo, Planck, 1906; von Laue, 1911), usa a teoria do eletromagnetismo de Maxwell (veja a Seção 3.1). No entanto, como Einstein observou mais tarde (1935), a equivalência massa-energia é um resultado que deve ser independente de qualquer teoria que descreve uma interação física específica. Esta é a razão principal que levou os físicos a procurarem derivações "puramente dinâmicas", ou seja, derivações que invocam somente conceitos mecânicos, tais como "energia" e "momento", e os princípios que os regem (veja a Seção 3.2).

\subsection{Derivações de $E=m c^{2}$ que usam a teoria de Maxwell}

A derivação original de Einstein da equivalência massa-energia é a mais conhecida neste grupo. Einstein começa com o seguinte experimento mental: um corpo em repouso (em algum referencial inercial) emite dois pulsos de luz de energia igual em direções opostas. Einstein então analisa este "ato de emissão" a partir de outro referencial inercial, que está num estado de movimento uniforme em relação ao primeiro. Nesta análise, Einstein utiliza a teoria de Maxwell do eletromagnetismo para calcular as propriedades físicas dos pulsos de luz (tal como a sua intensidade) no segundo referencial inercial. Comparando as duas descrições do "ato de emissão", Einstein chega ao seu resultado célebre: "a massa de um corpo é uma medida do seu conteúdo de energia; se a energia muda de $L$, a massa muda no mesmo sentido de $L / 9 \times 10^{20}$, sendo a energia medida em ergs, e a massa, em gramas" (1905b, p. 71; 2001, p. 
186). Uma derivação semelhante, usando o mesmo experimento mental, mas apelando para o efeito Doppler, foi dada por Langevin (1913) (veja a discussão sobre a inércia da energia em Fox, 1965, p. 8).

Alguns filósofos e historiadores da ciência afirmam que a primeira derivação de Einstein é falaciosa. Por exemplo, em O Conceito de Massa, Jammer diz: "É um incidente curioso na história do pensamento científico que a derivação do próprio Einstein da fórmula $E$ $=m c^{2}$, conforme publicado em seu artigo na Annalen der Physik, era basicamente falacioso [...] o resultado de uma petição de princípio, onde a demonstração pressupõe a conclusão" (Jammer, 1961, p. 177). Segundo Jammer, Einstein pressupõe implicitamente o que ele está tentando provar, ou seja, que se um corpo emite uma quantidade de energia $L$, sua massa inercial irá diminuir por um valor $\Delta m=L / c^{2}$. Jammer também acusa Einstein de pressupor a expressão da energia cinética relativística de um corpo. Se Einstein fez essas hipóteses, ele seria culpado de petição de princípio. Recentemente, no entanto, Stachel \& Torretti (1982) mostraram de forma convincente que o argumento de Einstein (1905b) é correto. Eles observam que Einstein de fato deriva a expressão para a energia cinética de um "elétron" (ou seja, uma partícula sem estrutura com uma carga), em seu artigo (1905a) anterior. No entanto, Einstein em lugar algum utiliza esta expressão na derivação (1905b) da equivalência massaenergia. Stachel \& Torretti também mostram que os críticos de Einstein ignoram dois passos cruciais que são suficientes para tornar a derivação de Einstein correta, já que não é preciso assumir que $\Delta m=L / c^{2}$.

A conclusão adicional de Einstein de que "a massa de um corpo é uma medida do seu conteúdo de energia" (1905b, p. 71) não se segue, a rigor, de seu argumento. Como Torretti (1996) e outros filósofos e físicos têm observado, o argumento de Einstein (1905b) permite a possibilidade de que, uma vez que a quantidade de energia de um corpo tenha sido utilizada na totalidade (e subtraída da massa, usando a relação de equivalência massa-energia), o restante seja diferente de zero. Em outras palavras, é apenas uma hipótese no argumento de Einstein (1905b) e, de fato, em todas as derivações de $E=m c^{2}$ na relatividade restrita, que nenhuma "matéria exótica" existe que não seja conversível em energia (ver Ehlers, Rindler \& Penrose, 1965, para uma discussão sobre este ponto). No entanto, experimentos de aniquilação partícula-antipartícula em física atômica, que foram observadas pela primeira vez décadas após 1905, apoiam fortemente "a intrépida extrapolação de Einstein” (Torretti, 1996, p. 112). 


\subsection{Derivações puramente dinâmicas de $E=m c^{2}$}

Derivações puramente dinâmicas de $E=m c^{2}$ tipicamente procedem por análise de uma colisão inelástica, a partir do ponto de vista de dois referenciais inerciais em um estado de movimento relativo (o referencial do centro de massa e um referencial inercial movendo-se com uma velocidade relativa $v$ ). Um dos primeiros trabalhos que parece seguir esta abordagem é o de Perrin (1932). De acordo com Rindler \& Penrose (1965), a derivação de Perrin foi baseada, em grande parte nas "elegantes" palestras de Langevin, que foram ministradas no Collège de France, em Zurique, por volta de 1922. O próprio Einstein deu uma derivação puramente dinâmica (Einstein, 1935), embora ele não mencione nem Langevin nem Perrin. A derivação mais abrangente desse tipo foi dada por Ehlers, Rindler \& Penrose (1965). Mais recentemente, uma versão puramente dinâmica do experimento mental original de Einstein (1905b), onde as partículas que são emitidas não são fótons, foi dada por Feigenbaum \& Mermin (1988).

Derivações deste grupo são distintivas porque demonstram que a equivalência massaenergia é uma consequência das alterações na estrutura do espaço-tempo trazidas pela teoria da relatividade restrita. A relação entre massa e energia é independente da teoria de Maxwell ou qualquer outra teoria que descreva uma interação física específica. Podemos ter um vislumbre disto notando que para obter $E=m c^{2}$ analisando uma colisão, é preciso primeiro definir o momento relativístico $\left(\boldsymbol{p}_{\text {rel }}\right)$ e energia cinética relativística $\left(T_{\text {rel }}\right)$, uma vez que não se pode usar as velhas noções newtonianas de momento e energia cinética. Na própria derivação puramente dinâmica de Einstein (1935), mais da metade do artigo é dedicado a encontrar as expressões matemáticas que definem $\boldsymbol{p}_{\text {rel }}$ e $T_{\text {rel. }}$ Todo esse trabalho é necessário para se chegar a estas expressões por duas razões. Primeiro, as alterações na estrutura do espaço-tempo devem ser incorporadas nas definições das grandezas relativísticas. Em segundo lugar, $\boldsymbol{p}_{\text {rel }}$ e $T$ rel devem ser definidos de modo que sejam reduzidos às suas contrapartidas newtonianas no limite adequado. Este último requisito garante, com efeito, que a relatividade restrita herdará o sucesso empírico da física newtoniana. Uma vez que as definições de $\boldsymbol{p}_{\text {rel }}$ e $T_{r e l}$ são obtidas, a derivação de equivalência massa-energia é direta. (Para uma discussão mais detalhada da derivação de Einstein de 1935, ver Flores, 1998.)

Finalmente, podemos agora entender porque nenhuma interpretação da equivalência massa-energia pode explicar por que a massa de repouso dos constituintes de um sistema físico contribui para a energia de repouso deste sistema, ou porque a energia dos constituintes 
contribui para a massa de repouso do sistema. Dadas as alterações na estrutura do espaçotempo impostas pela relatividade restrita, e tendo em conta as definições de grandezas dinâmicas que se adotam (por razões bem motivadas), pode-se certamente derivar a equivalência massa-energia a partir da relatividade restrita. Tal derivação, entretanto, só pode mostrar que a massa é equivalente à energia, no sentido em que temos nos esforçado para elaborar acima. Tal derivação, em outras palavras, é uma boa candidata para o que Kitcher familiarmente chama de explicação "de cima para baixo" ["top-down" explanation]. Para uma discussão mais aprofundada deste ponto, ver Flores $(1999,2005)$.

\section{Verificação experimental da equivalência massa-energia}

Cockcroft \& Walton (1932) são rotineiramente creditados com a primeira verificação experimental da equivalência massa-energia. Cockcroft \& Walton examinaram uma variedade de reações onde diferentes núcleos atômicos são bombardeados por prótons. Eles concentraram-se principalmente no bombardeamento de ${ }^{7} \mathrm{Li}$ por prótons (isto é, a reação $\underline{5}$ acima).

Em seu famoso artigo, Cockcroft \& Walton notaram que a soma de massas de repouso do próton e do núcleo de lítio (ou seja, os reagentes) foi de 1,0072 + 7,0104 = 8,0176 u (unidade de massa atômica). No entanto, a soma das massas de repouso das duas partículas $\alpha$ (isto é, os produtos) foi 8,0022 u. Assim, era como se uma quantidade de massa de 0,0154 u tivesse "desaparecido" dos reagentes. Cockcroft \& Walton também observaram que a energia total (no referencial em que o núcleo ${ }^{7} \mathrm{Li}$ está em repouso) para os reagentes foi de $125 \mathrm{KeV}$ (milhares de elétron-volts). No entanto, observaram que a energia cinética total das partículas $\alpha$ era 17,2 MeV (milhões de elétron-volts). Assim, era como se uma quantidade de energia de cerca de $17 \mathrm{MeV}$ tivesse "aparecido" na reação.

Referindo-se implicitamente à equivalência entre massa e energia, Cockcroft \& Walton, em seguida, simplesmente afirmam que uma massa de 0,0154 u "é equivalente a uma liberação de energia de $(14,3 \pm 2,7) \times 10^{6}$ Volts" (p. 236). Eles então sugerem implicitamente que este valor inferido para a energia cinética das duas partículas $\alpha$ resultantes é consistente com o valor observado para a energia cinética das partículas $\alpha$. Cockcroft \& Walton concluem que "as energias observadas das partículas $\alpha$ são consistentes com a nossa hipótese" (pp. 236237). A hipótese que eles estavam testado, entretanto, não é equivalência massa-energia, mas que quando o núcleo de ${ }^{7} \mathrm{Li}$ é bombardeado com prótons, o resultado são duas partículas $\alpha$. 
Como Stuewer (1993) sugeriu, Cockcroft \& Walton usam a equivalência massaenergia para confirmar sua hipótese sobre o que acontece quando ${ }^{7} \mathrm{Li}$ é bombardeado por prótons. Assim, não parece que devemos considerar este experimento como uma confirmação de $E=m c^{2}$. No entanto, se tomarmos algumas das outras evidências que Cockcroft \& Walton fornecem a respeito da identificação dos produtos na reação (모) como sendo suficientes para comprovar que os produtos são de fato partículas $\alpha$, então podemos interpretar este experimento como uma confirmação da equivalência massa-energia, que é como este experimento é frequentemente relatado na literatura física.

Muito mais recentemente, Rainville et al. (2005) publicaram os resultados do que eles

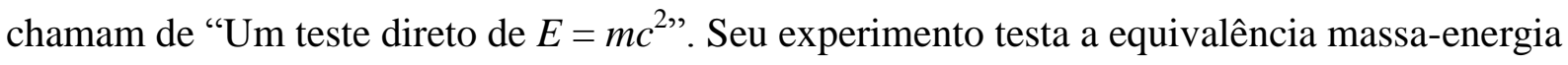
"diretamente", comparando a diferença nas massas de repouso, em uma reação de captura de nêutrons, com a energia dos raios gama $(\gamma)$ emitidos. Especificamente, Rainville et al. examinam duas reações, uma envolvendo a captura de nêutrons por enxofre $(S)$, a outra envolvendo a captura de nêutrons por silício $(\mathrm{Si})$ :

$$
\begin{gathered}
n+{ }^{32} S \rightarrow{ }^{33} S+\gamma \\
n+{ }^{28} \mathrm{Si} \rightarrow{ }^{29} \mathrm{Si}+\gamma
\end{gathered}
$$

Nestas reações, quando o núcleo de um átomo (neste caso tanto ${ }^{32} \mathrm{~S}$ quanto ${ }^{28} \mathrm{Si}$ ) captura o nêutron, um novo isótopo é criado em um estado excitado. Ao retornar ao seu estado fundamental, o isótopo emite um raio- $\gamma$. De acordo com a equação de Einstein, a diferença nas massas de repouso do núcleo mais o nêutron, por um lado, e do novo isótopo no seu estado fundamental, de outro lado, deve ser igual à energia do fóton emitido. Assim, Rainville et al. testam $\Delta E=\Delta m c^{2}$ fazendo medições muito acuradas da diferença de massa de repouso e da frequência e, portanto, da energia, do fóton emitido. Rainville et al. relatam que suas medições mostram que a equação de Einstein obtém uma precisão de pelo menos 0,00004\%.

\section{Conclusão}

Neste artigo, apresentamos uma interpretação mínima da equivalência massa-energia amplamente sustentada tanto por físicos quanto por filósofos. Também apresentamos uma variedade de interpretações filosóficas da equivalência massa-energia, algumas das quais vão além da interpretação mínima, com a qual começamos. Ao longo do caminho, apresentamos os méritos e deméritos de cada interpretação. Também apresentamos um breve histórico de derivações da equivalência massa-energia, para enfatizar que a equivalência entre massa e 
energia é um resultado direto de alterações na estrutura do espaço-tempo imposta pela relatividade restrita. Finalmente, discutimos breve e bastante seletivamente a confirmação empírica da equivalência massa-energia.

\section{Bibliografia}

Baierlein, R. (2007), “Does nature convert mass into energy?” Am. J. Phys., 75(4): 320-325.

Bondi, H. \& C.B. Spurgin (1987), “Energy has mass,” Phys. Bull., 38: 62-63.

Cockcroft, J. D. \& E. T. Walton (1932), "Experiments with high velocity positive ions (II) The disintegration of elements by high velocity protons," Proc. Roy. Soc. London, A137: 229-242.

Eddington, A. (1929), Space, Time, and Gravitation, London: Cambridge University Press, publicado originalmente em 1920.

Ehlers, J., W. Rindler, \& R. Penrose (1965), "Energy conservation as the basis of relativistic mechanics II," Am. J. Phys., 35: 995-997.

Einstein, A. (1905a), "On the electrodynamics of moving bodies," in A. Einstein et al. (1952), pp. 35-65. Tradução para o português: "Sobre a eletrodinâmica dos corpos em movimento", in Stachel (2001), pp. 143-182.

- (1905b), "Does the inertia of a body depend upon its energy content?" in A. Einstein et al. (1952), pp. 69-71. Tradução para o português: "A inércia de um corpo depende de seu conteúdo de energia?”, in Stachel (2001), pp. 183-186.

—. (1919), "What is the theory of relativity?" in A. Einstein (1982), pp. 227-232.

—. (1935), "Elementary derivation of the equivalence of mass and energy," Am. Math. Soc. Bul., 41: 223-230.

—. (1982), Ideas and Opinions, New York: Crown Publishers Inc.

Einstein, A., H.A. Lorentz, H. Minkowski \& H. Weyl (1952), The Principle of Relativity, trad. W. Perrett \& G.B. Jeffery, New York: Dover. Orig. 1917. Tradução para o português: O Princípio da Relatividade, trad. M.J. Saraiva, Lisboa: Gulbenkian, 1983.

Einstein, A. \& L. Infeld (1938), The Evolution of Physics, New York: Simon and Schuster. Tradução para o português: A Evolução da Física, trad. G. Rebuá, Rio de Janeiro, Zahar, 1962.

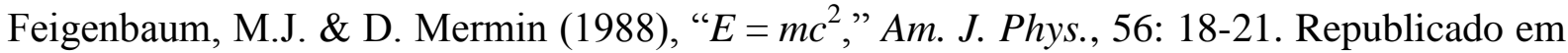
Mermin, D., Boojums All the Way Through, New York: Cambridge University Press, pp. 266-80. 
Flores, F. (1998), “Einstein's 1935 derivation of $E=m c^{2}$," Stud. Hist. Phil. Mod. Phys., 29(2): 223-243.

- (1999), "Einstein's theory of theories and types of theoretical Explanation," International Studies in the Philosophy of Science, 13(2): 123-134

—. (2005), "Interpretations of Einstein's equation $E=m c^{2}$," International Studies in the Philosophy of Science, 19(3): 245-260.

Fox, J. G. (1965), “Evidence against emission theories,” Am. J. Phys., 33(1): 1-17.

Jammer, M. (1961), Concepts of Mass in Classical and Modern Physics, Cambridge, MA: Harvard University Press.

Lange, M. (2001), “The most famous equation,” Journal of Philosophy, 98: 219-238.

—. (2002), An Introduction to the Philosophy of Physics, Oxford: Blackwell.

Langevin, P. (1913), "L'inertie de l'énergie et ses conséquences", Journal de Physique Théorique et Appliqueé (series 4), 3: 553-591.

Minkowski, H. (1908), “Space and time” in A. Einstein et al. (1952), pp. 75-91.

Perrin, F. (1932), La Dynamique Relativiste et l'Inertie de l'Energie. (Coleção Actualities Scientifiques et Industrielles, 41, 19 pgs.), Paris: Hermann.

Rainville, S. et al. (2005), “A direct test of $E=m c^{2}, "$ Nature, 438: 1096-1097.

Rindler, W. (1977), Essential Relativity, New York, N.Y.: Springer-Verlag.

Stachel, J. (org.) (2001), O Ano Miraculoso de Einstein, trad. A.C. Tort, Rio de Janeiro: Editora UFRJ.

Stachel, J. \& R. Torretti (1982), "Einstein's first derivation of mass-energy Equivalence," Am. J. Phys., 50(8): 760-761.

Stuewer, R.H. (1993), "Mass-energy and the neutron in the early thirties," Sci. Context, 6: 195-238.

Taylor, E.F. \& J.A. Wheeler (1992), Spacetime Physics, San Francisco: W. H. Freeman.

Torretti, R. (1996), Relativity and Geometry, New York: Dover.

Zahar, E. (1989), Einstein's Revolution: A Study in Heuristic, La Salle: Open Court. 